MAGNETIC NANOPARTICLES

\section{Self-assembly at the limit}

Proc. Natl Acad. Sci. USA

http://doi.org/885 (2015)

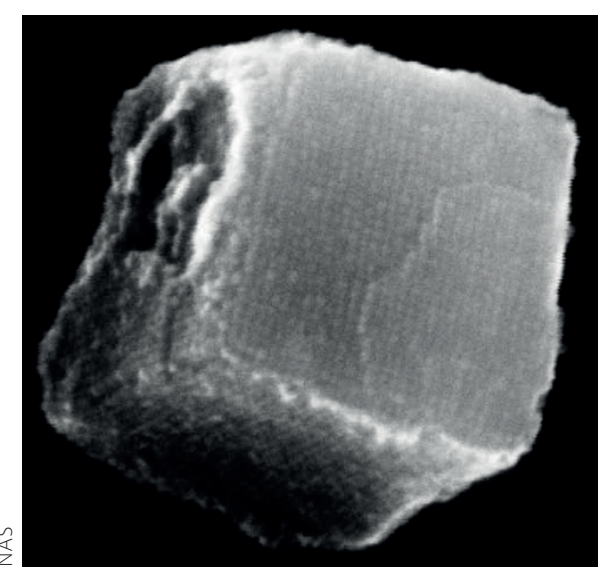

Self-assembly is a key concept in nanotechnology and comes in a variety of forms. One approach involves the spontaneous assembly of magnetic nanoparticles in the presence of an external magnetic field. There is, however, a size limit to the approach, which emerges as the nanoparticles get smaller and their magnetic moment decreases. Ingo Rehberg, Stephan Förster and colleagues have now shown that nanoparticles at this size limit can self-assemble in solution to form regular one-, two- and three-dimensional structures.

The researchers - who are based at the University of Bayreuth, the University Duisburg-Essen and the European Synchrotron Radiation Facility in
Grenoble - examined spherical and cube-shaped iron oxide nanoparticles that were stabilized by oleic acid and had diameters of less than $15 \mathrm{~nm}$. The spherical nanoparticles did not form regular structures in the presence of a magnetic field, but the cube-shaped nanoparticles assembled into chains, monolayer sheets and large cuboids, all with almost perfect internal order. These assemblies are created because the magnetic dipoles of the nanoparticles are aligned by the magnetic field, which leads to an attractive interaction that forces the nanoparticles into intimate contact; short-range van der Waals interactions then drive the nanoparticles into a regular face-to-face attachment, which is stable after the magnetic field is removed. For the spherical nanoparticles, it is thought that the short-range attractive interactions are much weaker and therefore assemblies cannot be stabilized.

Förster and colleagues also show that cube-shaped nanoparticles smaller than $7.5 \mathrm{~nm}$ cannot form assemblies.

OV

\section{PLASMONICS \\ Graphene crumpling \\ Nano Lett. 15, 7684-7690 (2015)}

In surface-enhanced Raman spectroscopy (SERS), Raman scattering is strongly amplified by placing molecules in close proximity to a metal surface. Surface roughening is generally thought of as the simplest way to improve SERS sensitivity, but a variety of more precise methods have been explored including decorating substrates with metal nanoparticles and using twodimensional hybrid structures composed

\title{
WATER CLUSTERS
}

\section{High-speed evaporation Angew. Chem. Int. Ed. http://doi.org/f3gw9r (2015)}

Water underpins the vast majority of biological and atmospheric phenomena. However, while the properties of bulk water are reasonably well understood, clusters of water molecules, which are usually involved in the onset of such phenomena, are relatively unexplored because they are much harder to study. Michel Farizon and colleagues at the University of Lyon, the University of Grenoble and Leopold Franzens University have now developed a method to measure the speed distribution of water molecules as they evaporate from clusters as small as two to eight molecules.

The researchers carefully selected their clusters based on mass, and then shot an argon atom at them. The energy of the impact gets redistributed among the $\mathrm{O}-\mathrm{H}$ oscillators of the water cluster, causing one of the molecules to evaporate. A detector then measures the speed and orientation of this leaving molecule and meaningful statistics are obtained by repeating the experiment multiple times.

Farizon and colleagues observe that the velocity distribution of evaporated water molecules is composed of a low- and a high-speed component. The low-speed molecules are expected from a complete redistribution of the impact energy among the vibrational modes of the cluster. The high-speed evaporation (about $30 \%$ of cases) occurs before all of the energy has reached a Maxwell-Boltzmann distribution. Such a non-ergodic chemical regime is fundamentally different from that of bulk water or even larger clusters.

of metal nanoparticles on graphene. SungWoo Nam and colleagues at the University of Illinois at Urbana-Champaign have now shown that SERS enhancements can be achieved by simply changing the dimensionality of such graphene structures.

The researchers began by fabricating a graphene film decorated with gold nanoparticles. This was transferred onto a polymer substrate that shrinks when heated, which allowed crumpled graphenenanoparticle hybrid structures to be created. The three-dimensional graphene structures could then be transferred to a variety of arbitrarily shaped curved surfaces. Compared with similar two-dimensional hybrid systems, the crumpled substrates offer a one order of magnitude stronger Raman signal. Nam and colleagues also suggest that further enhancement could potentially be achieved by tuning the size and spacing between the nanoparticles.

\section{NANOMEDICINE}

\section{Nanoworm protection \\ ACS Nano http://doi.org/9c3 (2015)}

Engineered nanoparticles are of potential use in biomedical applications such as magnetic resonance imaging and drug delivery, but typically offer limited haemocompatibility. This means that, when intravenously injected, the nanoparticles are recognized by the immune system and mechanisms are put in place to reduce or completely eliminate their presence. Dmitri Simberg and colleagues at the University of Colorado, Jilin University, and the University of Copenhagen have now used magnetic nanoparticles to explore immune recognition mechanisms and identify ways to mitigate their effects.

The researchers examined the interaction between blood leukocytes, which are cells known to be involved in intercepting pathogens, and superparamagnetic iron oxide nanoparticles that had a worm-like shape and were coated with dextran chains (referred to as 'nanoworms'). Uptake of these nanoworms by leukocytes in both mice and human blood was found to be dependent on the complement system of proteins. Haemocompatibility of the nanoworms could, therefore, be improved by using inhibitors of the complement system. Simberg and colleagues also found that haemocompatibility could be improved through crosslinking and hydrogelation of the dextran on the surface of the nanoworms.

Written by Olga Bubnova, Alberto Moscatelli and Owain Vaughan. 